Reprod. Nutr. Dévelop., 1986, 26 (2 B), 583-588.

\title{
Utilisation des produits terminaux de la digestion par la mamelle chez la vache laitière
}

\author{
H. RULQUIN
}

avec la collaboration technique de Jeanne FLECHET, Renée LEFAIVRE, A. OLLIER et Claire SORNET

Station de Recherches sur la Vache laitière, I.N.R.A. Saint-Gilles, 35590 L'Hermitage, France.

Summary. Use of the end-products of digestion by the udder of the dairy cow.

Relations between mammary arterio-venous (AV) differences and arterial level were studied for glucose, acetate and beta-hydroxybutyrate on 172 samples and for blood essential amino acids on 20 samples, pooled by day and by cow. The data were obtained from 32 experiments on 5 cows eating various diets (Rulquin, 1981, 1983). AV differences in acetate and beta-hydroxybutyrate were highly related to the arterial levels $(R=0.97$ and 0.86 ) and averaged 74 and $41 \%$ of these levels (fig. 1). On the other hand, the AV difference in glucose was poorly $(R=0.5)$ related to the arterial levels.

Among the essential amino acids, only AV differences in phenylalanine, leucine, arginine, isoleucine and histidine were linearly related to the arterial levels $(R=0.78,0.73$, $0.68,0.63,0.60$ ) (fig. 2). AV differences in lysine, methionine, valine and threonine were more related to the AV differences of other amino acids than to the arterial levels (fig. 3 ). These results have been discussed in connection with different mammary metabolic transport systems and with the ability to modify mammary nutrient input by dietary manipulation.

\section{Introduction.}

Un accroissement de la productivité des animaux laitiers peut être obtenu par une amélioration de l'efficacité de l'utilisation des nutriments pour la synthèse du lait consécutive à une modification de leur captation par la mamelle (Baldwin et al., 1980 ; Bauman et al., 1985). En pratique, cela nécessite de pouvoir agir, sur les différences artério-veineuses mammaires et (ou) le débit sanguin, par le biais de l'alimentation, ou de l'administration d'agents altérant le métabolisme. Les possibilités de modifier le débit sanguin mammaire sont mal connues, mais envisageables avec, notamment, l'administration d'hormone de croissance (Mepham, 1982). Les différences artério-veineuses mammaires de certains métabolites peuvent être altérées en modifiant leurs taux artériels (Blandwin et al., 1980). Nous avons complété ce dernier point en étudiant chez la vache laitière, sur un nombre important de mesures et dans des conditions d'alimentations variées, les relations existant entre les différences artério-veineuses mammaires (différences AV) et les taux artériels des principaux nutriments. 


\section{Matériel et méthodes.}

Cette étude réunit toutes les mesures de différences AV mammaires réalisées jusqu'ici dans notre laboratoire sur des vaches laitières dans des conditions diverses d'alimentation énergétique et azotée. Ces mesures ont été effectuées à l'aide de cathéters implantés dans la carotide (chirurgicalement amenée en position sous-cutanée) et dans la veine sous-cutanée abdominale. Elles ont été réalisées au cours de deux essais :

- un essai comportant 8 cinétiques (prélèvements à $7 \mathrm{~h}, 10 \mathrm{~h}, 13 \mathrm{~h}, 16 \mathrm{~h}, 19 \mathrm{~h}$, $22 \mathrm{~h}, 1 \mathrm{~h}, 4 \mathrm{~h})$ qui ont été effectuées sur 2 vaches en $7 \mathrm{e}, 11^{\mathrm{e}}, 19^{\mathrm{e}}$ et $22^{\mathrm{e}}$ semaine de lactation. Ces vaches étaient alimentées suivant leurs besoins et recevaient successivement, suivant un schéma en inversion, un régime comprenant 70 ou $30 \%$ d'aliments concentrés qui étaient distribués en 2 ou 8 fois par jour (Rulquin, 1981).

- un essai comprenant 24 cinétiques (prélèvements à $7 \mathrm{~h}, 8 \mathrm{~h} 30,10 \mathrm{~h}, 13 \mathrm{~h}$, $16 \mathrm{~h}, 2$ jours de suite) qui ont été réalisées sur 3 vaches en $8^{\mathrm{e}}, 12^{\mathrm{e}}, 16^{\mathrm{e}}, 20^{\mathrm{e}}$ semaine de lactation. Ces vaches ont été alimentées suivant un schéma en carré de Youden $4 \times 3$ croisant 2 niveaux énergétiques ( 85 et $110 \%$ des besoins) et 2 niveaux azotés (70 et $100 \%$ des besoins). L'écart entre les niveaux énergétiques était créé par l'infusion ruminale $(2,4$ ou 23,8 moles/j) d'un mélange d'acides gras volatils et entre les niveaux azotés par infusion duodénale $(0 \mathrm{ou} 500 \mathrm{~g} / \mathrm{j})$ de caséinate de sodium (Rulquin, 1983). En outre, le mélange d'acides gras volatils contenait $56 \%$ d'acide propionique de façon à simuler le type de fermentations survenant avec des régimes riches en aliments concentrés.

Le détail des conditions expérimentales et des méthodes de dosage de l'acétate, du bêta-hydroxybutyrate, du glucose et des acides aminés libres sanguins a été décrit précédemment (Rulquin, 1981, 1982, 1983).

Le nombre total des déterminations de différences AV est de 172 pour le glucose, l'acétate, le bêta-hydroxybutyrate et de 20 pour les acides aminés libres du sang car leur dosage n'a été effectué que sur des échantillons regroupant les points d'une ou deux cinétiques.

\section{Résultats.}

Les différences AV d'acétate et de bêta-hydroxybutyrate sont liées de façon très étroite (corrélation $(R)=0,97$ et 0,86 ) aux taux artériels (fig. 1). Elles repré-
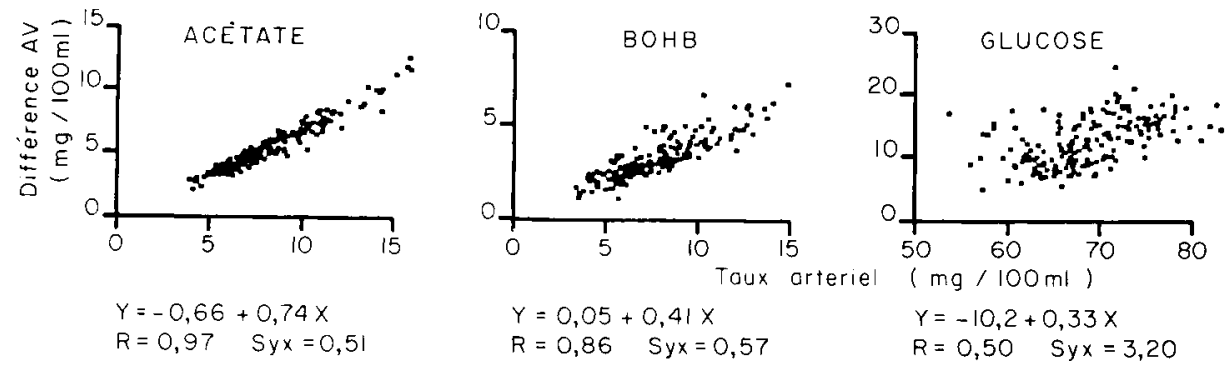

FIG. 1. - Relations entre les différences artério-veineuses et les taux artériels des nutriments énergétiques. 
sentent respectivement $74 \pm 2$ et $41 \pm 2 \%$ des taux arrivant à la mamelle. En moyenne les différences $A V$ de glucose atteignent $33 \pm 4 \%$ des taux afférents, mais leurs variations ne sont que faiblement liées à celles des taux artériels $(R=$ $0,5)$ (fig. 1). Cette relation est par ailleurs significativement $(P<0,05)$ affectée par la nature du régime. En effet, elle correspond à :

- différence $A V=-25+0,53 \times$ taux artériel $(n=81 ; R=0,64$; Syx $=2,9$ ) pour des régimes riches en aliments concentrés et,

- différence $A V=-2,7+0,24 \times$ taux artériel $(n=89 ; R=0,46 ;$ Syx $=3$ ) pour des régimes riches en fourrages.

Les variations des différences $A V$ des acides aminés indispensables tels que la phénylalanine, la leucine, I'arginine, I'isoleucine et l'histidine (fig. 2) sont linéairement dépendantes $(P<0,05)$ de leurs taux artériels $(R=0,78 ; 0,73 ; 0,68$; $0,63 ; 0,60)$. Ces différences AV représentent respectivement, $57 \pm 11,25 \pm 6$, $26 \pm 7,18 \pm 5$ et $21 \pm 6 \%$ du taux de chacun des acides aminés arrivant à la mamelle.

Les différences $A V$ de valine, méthionine, thréonine et lysine sont pratiquement indépendantes de leurs taux dans l'artère (fig. 2). Aussi le taux de captation
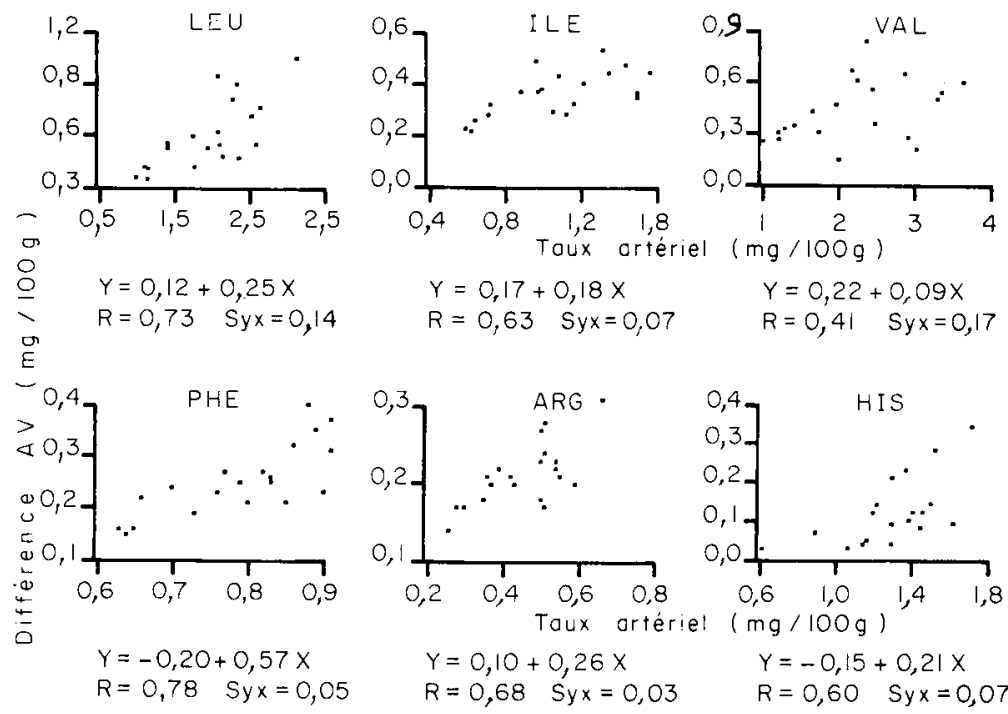

$$
\begin{aligned}
& Y=0,17+0,18 x \\
& R=0,63 \quad \text { Syx }=0,07
\end{aligned}
$$

$Y=0,22+0,09 x$

$R=0,41 \quad$ Syx $=0,17$
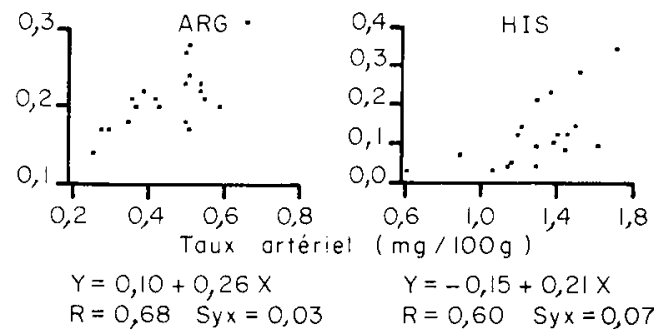

$$
\begin{aligned}
& Y=-0,15+0,21 x \\
& R=0,60 \text { Syx }=0,07
\end{aligned}
$$
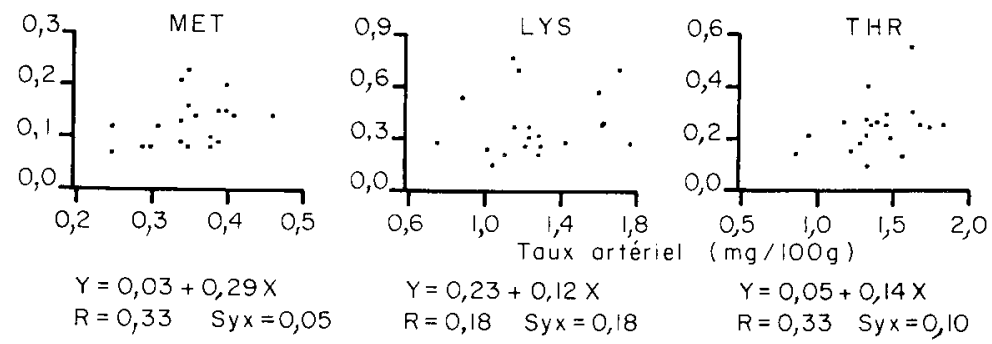

FIG. 2. - Relations entre les différences artério-veineuses et les taux artériels des acides aminés indispensables. 
de ces acides aminés dans le sang artériel (en moyenne respectivement, 9, 29, 14 et $12 \%$ ) est très variable. Les différences AV de lysine sont étroitement liées à celles d'histidine (fig. 3), qui est un acide aminé utilisant le même transporteur spécifique des acides aminés basiques (Christensen, 1977). Pour la méthionine, la thréonine et la valine, acides aminés ayant plusieurs systèmes de transport, ces relations (fig. 3) s'établissent essentiellement avec les acides aminés transportés par le système $L$ c'est à dire la leucine, l'isoleucine et la phénylalanine.

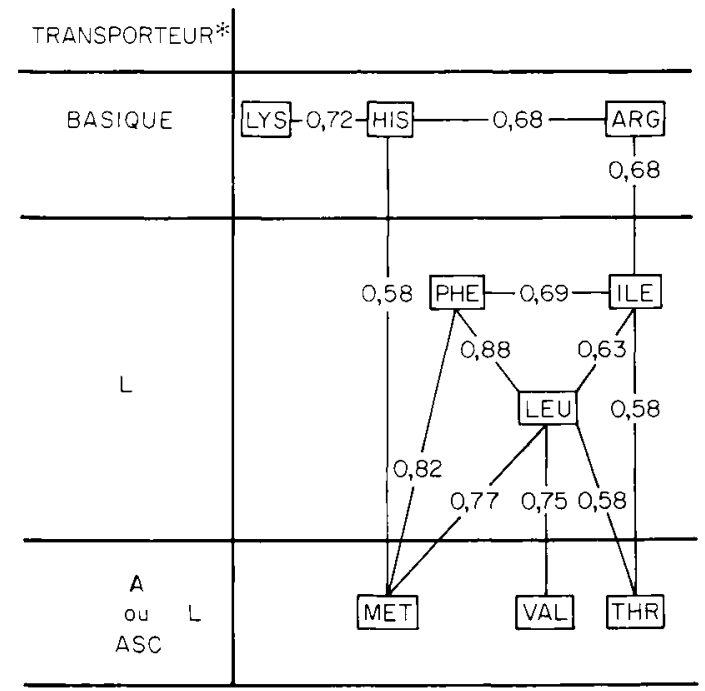

FIG. 3. - Corrélations entre les différences AV des acides aminés indispensables.

(Les corrélations indiquées sont significatives à $P<0,01$. Classification de Christensen (1977)).

\section{Discussion.}

L'importance des captations d'acétate et de bêta-hydroxybutyrate par la mamelle et leur quasi proportionnalité avec les taux artériels correspondent aux observations de Baldwin et al. (1980), tant que les taux artériels restent dans le domaine du physiologique. En effet, la captation du bêta-hydroxybutyrate diminue (Baldwin et al., 1980) pour des taux artériels de 2 à 3 fois plus élevés provenant de mesures effectuées par Kronfeld, Raggi et Ramberg (1968) sur des vaches acétonémiques ou à jeun.

La proportionnalité des différences $A V$ avec les taux circulants reflète le mécanisme de l'absorption de ces deux métabolites par la mamelle, qui comme pour le foie ou le muscle doit s'effectuer par diffusion passive (Madsen, 1983). Le prélèvement des ces nutriments par la mamelle peut être facilement altéré par l'alimentation, car il est possible de modifier leur taux artériel par son intermédiaire (Annison, Bickerstaffe et Linzell, 1974).

Ce n'est pas le cas du glucose en raison de l'indépendance, également observée par Baldwin et al. (1980), entre les variations des différences AV et des taux 
artériels. Cette indépendance pourrait traduire l'existence d'un système de transport du glucose au niveau de la mamelle, sur lequel des facteurs autres qu'alimentaires peuvent agir. Ainsi la captation du glucose diminue avec l'avancement de la lactation (Rulquin, 1981 ; Fleet et Mepham, 1985). Certaines hormones peuvent être à l'origine des modifications de la captation, c'est le cas des glucocorticoïdes (Kronfeld, 1982), mais pas de l'insuline (Laarveld, Christensen et Brockman, 1981).

Les liaisons linéaires entre les taux artériels et les différences AV d'isoleucine, de leucine, de phénylalanine correspondent aux observations effectuées intra vaches par Peeters et al. (1979) et intra brebis par Fleet et Mepham (1985). Ces liaisons sont peut-être dues au fait que ces 3 acides aminés sont transportés par un même système (système L) insensible aux hormones comme l'insuline (Guidotti et al., 1976). Les résultats concernant les autres acides aminés suggèrent qu'il existe des synergies et des antagonismes entre les différents systèmes de transporteurs d'acides aminés pouvant expliquer la variabilité importante de la captation mammaire de ces acides aminés. Ceci demande toutefois à être étudié sur un nombre de mesures plus important que celui sur lequel a été effectué la présente étude ou celles de Peeters et al., (1979) et de Fleet et Mepham (1985).

\section{Conclusions.}

Cette étude montre que l'alimentation peut directement modifier la captation mammaire d'une partie des précurseurs des matières grasses du lait, mais qu'elle n'a que des effets indirects sur la captation des autres nutriments. Afin d'en contrôler ses effets, des études plus approfondies sur les mécanismes de transport et leur régulation s'avèrent nécessaires.

11 Réunion du groupe Développement I.N.R.A., Montpellier, 22-24 mai 1985.

Remerciements. - Nous remercions vivement $M$. Lefaivre pour la réalisation des opérations chirurgicales, M. Pion et ses collaborateurs pour les dosages d'acides aminés.

\section{Références}

ANNISON E. F., BICKERSTAFFE R., LINZELL J. L., 1974. Glucose and fatty acid metabolism in cows producing milk of low fat content. J. agric. Sci. Camb., 82, 87-95.

BALDWIN R. L., SMITH N. E., TAYLOR J., SHARP M., 1980. Manipulating metabolic parameters to improve growth rate and milk secretion. J. anim. Sci, 51, 1416-1428.

BAUMAN D. E., Mc CUTCHEON S. N., STEINHOUR W. D., EPPARD P. J., SECHEN S. J., 1985. Sources of variation and prospects for improvement of productive efficiency in the dairy cow : A review. J. anim. Sci., 60, 583-592.

CHRISTENSEN H. N., 1977. Implications of the cellular transport step for amino acid metabolism. Nutr. Rev., 35, 129-133.

FLEET I. R., MEPHAM T. B., 1985. Mammary uptake of amino acids and glucose throughout lactation in Friesland sheep. J. Dairy Res., 52, 229-237.

GUIDOTTI G. G., BORGHETTI A. F., GAZZOLA G. C., TRAMACERE M., DALL'ASTA V., 1976. Insulin regulation of amino acid transport in mesenchymal cells from avian and mammalian tissues. Biochem. J., 160, 281-286. 
KRONFELD D. S., RAGGI F., RAMBERG C. F., 1968. Mammary blood flow and ketone body metabolism in normal, fasted and ketotic cows. Amer. J. Physiol., 215, 218-227.

KRONFELD D. S., 1982. Major metabolic determinants of milk volume, mammary efficiency and spontaneous ketosis in dairy cows. J. Dairy Sci, 65, 2204-2212.

LAARVELD B., CHRISTENSEN D. A., BROCKMAN R. P., 1981. The effect of insuline on net metabolism of glucose and amino acids by the bovine mammary gland. Endocrinology, 108, 2217 2221.

MADSEN A., 1983. The molecular basis of animal production. Metabolism in skeletal muscle cells. In RIIS P. M., Dynamic biochemistry of animal production (World Animal Science, A3), Elsevier Sci. Publ. B.V., 29-50.

MEPHAM T. B., 1982. Amino acid utilization by lactating mammary gland. J. Dairy Sci, 65, 287298.

PEETERS G., HOUVENAGHEL A., ROETS E., MASSART-LEËN A. M., VERBEKE R., DHONDT G., VERSHOOTEN F., 1979. Electromagnetic blood flow recording and balance of nutrients in the udder of lactating cows. J. anim. Sci., 48, 1143-1153.

RULQUIN H., 1981. Etude méthodologique sur la mesure des différences artérioveineuses mammaires chez la vache laitière. Reprod. Nutr. Dévelop., 21, 31-46.

RULQUIN H., 1982. Effets sur la digestion et le métabolisme des vaches laitières d'infusions d'acides gras volatils dans le rumen et de caséinate dans le duodénum. I. Production et digestion. Reprod. Nutr. Dévelop., 22, 905-921.

RULQUIN H., 1983. Effets sur la digestion et le métabolisme des vaches laitières d'infusions d'acides gras volatils dans le rumen et de caséinate dans le duodénum. II. Métabolisme général et mammaire. Reprod. Nutr. Dévelop., 23, 1029-1042. 